NYU-TH/00/06/05

June 1, 2000

\title{
On Sub-Millimeter Forces From Extra Dimensions
}

\author{
Gia Dvali, Gregory Gabadadze, Massimo Porrati \\ Department of Physics, New York University, New York, NY 10003
}

\begin{abstract}
We show that in theories with large extra dimensions forces mediated by a bulk dilaton and bulk gauge fields may be parametrically (exponentially) weaker than gravity due to the suppression of their wave-functions on a brane. This is the case when dilaton gets stabilized by certain strongly coupled dynamics on the brane, or the bulk gauge symmetries are spontaneously broken by the Higgs mechanism on the worldvolume. At distances smaller than the size of a largest extra dimension these particles produce the force-law which decreases with distance faster than highdimensional gravity. For a millimeter size extra dimensions predicted deviations are in the range which may be detected in sub-millimeter gravity measurements.
\end{abstract}




\section{Introduction}

Theories with low-scale quantum gravity provide an additional motivation to study the physics at sub-millimeter distances. In this framework the ordinary matter is localized on a brane which is embedded in space with $N$ large dimensions to which gravity can spread [1, 2]. As a result, the four-dimensional gravity measured at large distances is weak. These theories predict a deviation from Newtonian potential at distances below the maximal size of extra dimensions, $R<\mathrm{mm}$. The deviation can be measured in principle by upcoming experiments, provided that $R$ is somewhere in the range of a tenth of a micron or so [3, [1].

In the present paper we study the forces which, in addition to gravity, are present in the class of theories mentioned above. These forces can be tested and constrained by "table-top" experiments even for $R<<\mathrm{mm}$ [3, [1]. Examples of these are the forces mediated by some of the bulk fields. The bulk fields, by definition, can propagate in extra space and can couple to matter on the brane with the strength which is comparable to that of gravity. This statement is independent of the number and size of extra dimensions. The most widely discussed extra forces are those mediated by a dilaton and by bulk gauge fields. In the massless limit the coupling of their zero-modes to matter fields on a brane is suppressed by an universal volume factor

$$
\frac{1}{\sqrt{M_{\mathrm{Pf}}^{2+N} V_{N}}} .
$$

Here, $M_{\mathrm{Pf}}$ denotes the fundamental Planck constant of the $(4+N)$-dimensional theory, and $V_{N}$ stands for the volume of extra compactified space. It is assumed usually that these particles interact with matter fields with the coupling which is comparable to (in the case of a dilaton) or even stronger than (in case of gauge interactions) [2] the gravitational one. If the physics responsible for their masses originates from the brane, these particles acquire masses which are typically of order an inverse millimeter or less. Therefore, it is expected that at sub-millimeter distances they can mediated forces comparable or much stronger than gravity [2].

In the present note we argue that this lore is violated in certain cases:

- Interactions of bulk states with ordinary matter fields can be parametrically (exponentially) suppressed, so that forces mediated by these states can be considerably weaker than gravity;

- At distances less than the size of the extra dimension they can give rise to a force-law which differs from that of gravity.

The origin of these phenomena is quite simple. If the masses of the bulk states are induced due to interactions on a brane, then the wave-functions of the corresponding lowest modes are strongly suppressed in the brane worldvolume. In other words, as in ordinary quantum-mechanical systems, the corresponding wave-function "sees" 
the mass term induced on a brane as a repulsive potential and tends to avoid it. This is true for both a dilaton and bulk gauge fields. Concrete examples will be given in the subsequent sections. In the case of bulk gauge fields there is a simple physical interpretation of the effect. Let us discuss this interpretation first.

Consider $U_{B}(1)$ gauge symmetry in the bulk. We will be assuming that (some of the) standard model matter fermions are charged under this group. In particular, this may be a gauged $(B-L)$-symmetry or some other combination of lepton and baryon numbers. The corresponding "photon" $A_{B}$ can propagate in the bulk and can interact with the matter localized on the brane. If a zero-mode of the gauge field is massless, then there is an unbroken gauge symmetry in the effective 4D worldvolume theory with an effective gauge coupling constant given by [2]

$$
g_{\mathrm{eff}}=\frac{1}{\left(M_{\mathrm{Pf}} R\right)^{\frac{N}{2}}}=\frac{M_{\mathrm{Pf}}}{M_{P}},
$$

were we assume that a high-dimensional gauge coupling is of the order of unity in the $M_{\mathrm{Pf}}$-units . This is the strength by which an each Kaluza-Klein (KK) mode of the bulk gauge field $A_{B}$ couples to the $U_{B}(1)$-charged matter, in particular, the matter on a brane. The force mediated by these interactions between, say, two protons is related to their gravitational attraction as follows:

$$
\frac{F_{\text {gauge }}}{F_{\text {gravity }}} \propto \frac{M_{\mathrm{Pf}}^{2}}{m_{p}^{2}},
$$

were $m_{p}$ is the proton mass. For $M_{\mathrm{Pf}} \sim(1-10) \mathrm{TeV}$, this gives the force which is $\left(10^{6}-10^{8}\right)$ times bigger than that of gravity. Let us now suppose that the $U_{B}(1)$ symmetry is spontaneously broken by some Higgs field $\chi$ (not to be confused with the Standard Model Higgs) which condenses on a brane. A simple argument based on $4 \mathrm{D}$ gauge invariance suggest that the gauge field acquires the mass

$$
m_{\text {gauge }}=g_{\text {eff }}\langle\chi\rangle \text {, }
$$

and the gauge force will be screened at distances $r>>m_{\text {gauge }}^{-1}$. On the other hand, at distances $r<<m_{\text {gauge }}^{-1}$ charges are not screened and power-law gauge forces can be observed. One may expect, therefore, that the force at smaller distances will again be related to the gravitational force as in (3). However, as we will see, this expectation is violated if $\chi$ lives on "our" brane. In fact, even at distances $r<<m_{\text {gauge }}^{-1}$, the force may be exponentially weaker than gravity. The effect can be most easily understood by means of the analogy with superconductivity. As seen by a high-dimensional observer, the brane becomes superconducting, and the photon is repelled from its worldvolume due to the high-dimensional version of the Meissner effect. As a result, the coupling of the photon to fields localized inside the

\footnotetext{
${ }^{1}$ This is realized if, for instance, a 3 -brane is embedded in a worldvolume of a higher dimensional brane on which the dimensionfull gauge coupling is defined by the string scale.
} 
superconductor is suppressed by the strength of the condensate. This is an additional suppression, which in fact is absent for strictly massless high-dimensional fields, in particular for gravity. Therefore, the interaction mediated by a bulk gauge field on the brane is expected to be parametrically suppressed with respect to gravity. In the next two sections we will demonstrate this on concrete examples.

\section{Extra dimensions in the infinite volume limit}

Let us first consider the dilaton field. It enters the action in higher dimensional space as follows:

$$
M_{\mathrm{Pf}}^{2+N} \int d^{4+N} X \sqrt{|G|}\left(\mathcal{R}+G^{A B} \partial_{A} \Phi \partial_{B} \Phi+\ldots\right) .
$$

Here, $\mathcal{R}$ is the $(4+N)$-dimensional Ricci scalar, and ... stand for other possible terms and interactions. The dilaton field $\Phi$ interacts with the states which live in the brane worldvolume. This interaction in general takes the form:

$$
S_{\mathrm{int}}=\int d^{4} x \sqrt{|G(x, 0)|} \Phi(x, 0) \mathcal{O}(x),
$$

where $x$ denotes the $4 \mathrm{D}$ coordinates, $G(x, 0)$ is the induced metric on a brane, and $\mathcal{O}(x)$ denotes an operator of the brane worldvolume theory. An example of this operator would be the trace of the energy-momentum tensor of the worldvolume theory, $T_{\mu}^{\mu}(x)$. If the dilaton were massless, its exchange would produce scalar attraction of the gravitational strength, which would be phenomenologically unacceptable. Therefore, a dilaton should be massive in any physically viable scenario. Usually it is assumed that the dilaton mass comes from some non-perturbative dynamics which generates its potential. Let us ask what happens if this potential is generated due to the physics localized on our brane. The precise nature of this mass will be irrelevant for our analysis. We would like to investigate the potential mediated by the dilaton on a brane at the distances smaller than the compactification radius. Therefore, as a first step we will study one uncompactified extra dimension.

Suppose that the dilaton potential $V(\Phi)$ is generated on a brane which is located in extra space at the point $y=0$. Then the action for the dilaton can be written as follows:

$$
\frac{M_{\mathrm{Pf}}^{3}}{2} \int d^{4} x d y\left\{\partial_{A} \Phi \partial^{A} \Phi-V(\Phi) \delta(y)+\ldots\right\}
$$

In this expression, for sake of simplicity, we omit the gravitational part; it is assumed that gravity produces an appropriate classical background on which the dilaton field propagates (the dilaton background will be discussed below). The corresponding classical equation of motion for the dilaton takes the form:

$$
\partial_{B} \partial^{B} \Phi+\delta(y) \frac{d V}{d \Phi}=0
$$


The solution to this equation defines the classical dilaton background which we denote by $\Phi_{\mathrm{cl}}$. We are interested in fluctuations about this background. Therefore, we decompose the dilaton field as follows:

$$
\Phi=\Phi_{\mathrm{cl}}+\Delta \Phi
$$

The equation of motion for the fluctuations takes the form:

$$
\left(\partial_{B} \partial^{B}+\left.\delta(y) \frac{d^{2} V}{d \Phi^{2}}\right|_{\Phi_{\mathrm{cl}}}\right) \Delta \Phi=0 .
$$

The quantity $d^{2} V /\left.d \Phi^{2}\right|_{\Phi_{\mathrm{cl}}}$ can be thought of as the dilaton "mass" term on the brane?. In what follows we denote this quantity by $M \equiv d^{2} V /\left.d \Phi^{2}\right|_{\Phi_{\mathrm{cl}}}$. Thus, the dilaton fluctuations obey the following linearized equation:

$$
\left(\partial_{B}^{2}+M \delta(y)\right) \Delta \Phi(x, y)=0
$$

This reduces to a simple quantum-mechanical problem. Indeed, introducing the notations:

$$
\Delta \Phi(x, y)=e^{i p x} \Psi_{m}(y), \quad p^{2} \equiv p_{0}^{2}-\vec{p}^{2}=m^{2}
$$

we derive the following Schrödinger type equation:

$$
\left(-\partial_{y}^{2}+M \delta(y)\right) \Psi_{m}(y)=m^{2} \Psi_{m}(y) .
$$

This equation has a continuous tower of solutions with plane wave profiles in the extra dimension. The quantity which we will need below is the square of the wavefunction on the brane. This looks as follows:

$$
\left|\Psi_{m}(y=0)\right|^{2}=\frac{4 m^{2}}{4 m^{2}+M^{2}} .
$$

We see that the wave-functions of the light modes (which are most important at large distances) are suppressed by the factor $m^{2} / M^{2}$. The exchange of each mode mediates the Yukawa type force

$$
\frac{\exp (-m r)}{r}
$$

among the particles localized on the brane (here $r$ denotes a 3D distance on a brane). The net force is obtained by integrating over $m$. However, the leading effect comes only from the modes which are lighter than $M$. The wave functions of other states are unsuppressed on the brane and they produce the usual higher-dimensional

\footnotetext{
${ }^{2}$ Note that we use an unconventional normalization for the field $\Phi$.
} 
potential. The leading term in the potential mediated between two non-relativistic particles of masses $m_{1}$ and $m_{2}$ is equal to

$$
\begin{array}{r}
V(r) \simeq m_{1} m_{2} G_{5} \int_{0}^{M} \frac{\mathrm{e}^{-m r}}{r}\left|\Psi_{m}(y=0)\right|^{2} d m \\
\simeq \frac{4 m_{1} m_{2} G_{5}}{M^{2}} \int_{0}^{M} \frac{\mathrm{e}^{-m r}}{r} m^{2} d m \propto \frac{m_{1} m_{2} G_{5}}{M^{2} r^{4}}+\ldots
\end{array}
$$

Here, $G_{5}$ denotes the 5D Newton constant and dots stand for subleading corrections. We see a very strong additional suppression (compared to the 5D gravitational law) by a factor $M^{2} r^{2}>>1$. This indicates that dilaton-mediated force is much weaker than gravity.

Given the significance of this result it is instructive to understand it in a different approach. We can calculate the corresponding retarded Green function for the dilaton and then find the potential. The classical equation for the Green function looks as follows:

$$
\left(\partial_{A} \partial^{A}+M \delta(y)\right) G_{R}(x, y ; 0,0)=\delta^{(4)}(x) \delta(y)
$$

where $G_{R}(x, y ; 0,0)=0$ for $x_{0}<0$. The potential mediated by the dilaton on the $4 \mathrm{D}$ worldvolume of the brane is determined as:

$$
V(r)=\int G_{R}(t, \vec{x}, y=0 ; 0,0,0) d t
$$

where $r \equiv \sqrt{x_{1}^{2}+x_{2}^{2}+x_{3}^{2}}$. To find a solution of (17) let us turn to Fourier-transformed quantities with respect to the worldvolume four-coordinates $x^{\mu}$ :

$$
G_{R}(x, y ; 0,0) \equiv \int \frac{d^{4} p}{(2 \pi)^{4}} e^{i p x} \tilde{G}_{R}(p, y)
$$

Turning to Euclidean space the equation (17) takes the form:

$$
\left(\left(p^{2}-\partial_{y}^{2}\right)+M \delta(y)\right) \tilde{G}_{R}(p, y)=\delta(y) .
$$

Here $p^{2}$ denotes the square of an Euclidean four-momentum. The solution with boundary conditions appropriate for our problem takes the form:

$$
\tilde{G}_{R}(p, y)=\frac{1}{M+2 p} \exp (-p|y|)
$$

where $p \equiv \sqrt{p^{2}}=\sqrt{p_{4}^{2}+p_{1}^{2}+p_{2}^{2}+p_{3}^{2}}$. Using this expression and Eq. (18) one finds the following formula for the $4 \mathrm{D}$ dilaton-mediated potential:

$$
V(r) \propto \frac{1}{2 r}\left(-\frac{d}{d r}\right) \operatorname{Re}\left\{\exp \left(-\frac{i M r}{2}\right) E_{1}\left(-i \frac{M r}{2}\right)\right\} .
$$


Here, $E_{1}(z) \equiv \int_{z}^{\infty} e^{-t} d t / t$ denotes the exponential integral. For the large distances this gives

$$
V(r) \propto \frac{1}{M^{2} r^{4}}
$$

Therefore, we find the same $1 / r^{4}$ scaling of the potential at large distances. We conclude that the dilaton-mediated potential differs from the gravitational one due to the suppression of the dilaton wave-function on a brane.

So far we have studied the case when the dilaton potential $V(\Phi)$ is generated on a brane. It is conceivable that a kinetic term for a dilaton can also be induced on a brane (along the lines of Ref. [5]). In this case, the scaling laws of the force mediated by a dilaton would change. Indeed, let us assume that there is an additional kinetic term in (7) of the following form:

$$
M_{\mathrm{Pf}}^{3} \frac{\partial_{\mu} \Phi \partial^{\mu} \Phi}{2 \Lambda} \delta(y)
$$

where $\Lambda$ is some dimensionfull parameter. With this term in (7) we obtain the following expression for the Green function (21)

$$
\tilde{G}_{R}(p, y) \propto \frac{\Lambda}{p^{2}+2 \Lambda p+M \Lambda} \exp (-p|y|) .
$$

At short distances, $r<<\min \{1 / \sqrt{M \Lambda}, 1 / \Lambda\}$, the corresponding potential scales as $1 / r$. In the region $\max \{1 / \sqrt{M \Lambda}, 1 / \Lambda\}<<r<<1 / M$, the potential behaves as $1 / r^{2}$. Finally, at large distances $r>\max \{1 / \sqrt{M \Lambda}, 1 / M\}$ the potential scales as $1 / r^{4}$; this term is further modified by subleading corrections.

\section{Finite volume extra dimensions}

In the previous section we have treated the brane as a zero-thickness plane embedded in the infinite-volume extra dimension. Let us now study the case of a finite-thickness brane embedded in a finite volume extra space. We shell denote the brane thickness by $B$ and will assume that the brane is located at the center of an interval. In this framework the details of the interactions depend on the overlap between the transverse size on which the dilaton potential is supported on a brane, and the transverse widths of the domain on which matter fields are localized. Generically this overlap can be small. In what follows, we will assume for simplicity that ordinary matter is localized at a single point in the transverse space, i.e., we simply assume the delta-function type localization at $y=0$. As we mentioned above, the width of the transverse region on which the dilaton potential is supported will be some nonzero constant $B$ (one could think of a brane on which matter fields are localized, to be embedded into a wider brane on which the dilaton potential is 
supported). Qualitatively, this construction can be modeled by the following action

$$
\frac{M_{\mathrm{Pf}}^{3}}{2} \int d^{4} x d y\left\{\left(\partial_{A} \Phi\right)^{2}-M_{0}^{2} \delta_{R}(y, B) \Phi^{2}\right\}+\int d^{4} x \Phi(x, 0) T_{\mu}^{\mu}(x)+\ldots
$$

Here, as before, we omit gravitons and other terms concentrating on the dilaton field only. $\delta_{R}(y, B)$ is a bell-shaped function which determines that the mass term of the $\Phi$ field (or its potential) is spread over the transverse size $B$ of a "fat" brane 3. One can think of $\delta_{R}(y, B)$ as the smoothed Dirac's delta function with the unit hight and width $B$. Let us compactify the extra space on a circle of radius $\mathrm{R}$. We assume that $R$ is much bigger than the width $B$. Following the usual routine of compactification we decompose the field $\Phi$ in its $4 \mathrm{D}$ and extra dimensional parts:

$$
\Phi(x, y)=\sum_{n} \varphi_{n}(x) \Psi_{n}(y) .
$$

In what follows, we will retain for simplicity only the zero-mode; all other modes can be included analogously. Setting the normalization as

$$
\int_{0}^{2 \pi R} \Psi_{n}^{2}(y) d y=2 \pi R
$$

and rescaling the zero mode

$$
\varphi(x)=\frac{\sigma}{\sqrt{2 \pi R M_{\mathrm{Pf}}^{3}}}=\frac{\sigma}{\sqrt{M_{P}^{2}}}=\sqrt{G_{N}} \sigma,
$$

we get the canonically normalized Lagrangian for the massive scalar field $\sigma$

$$
\frac{1}{2}\left(\partial_{\mu} \sigma\right)^{2}-\frac{1}{2} m^{2} \sigma^{2}+\sqrt{\Psi^{2}(0) G_{N}} \sigma T_{\mu}^{\mu} .
$$

Here the dilaton (would be) zero-mode mass is $m^{2}=M^{2} \int \delta_{R}(y, B) d y / R$. The interaction of the $\sigma$ field with the matter is determined not by the Newton constant alone, but also by $\Psi(0)$. Thus, the effective interaction constant reads as follows:

$$
G_{\text {Dilaton }}=G_{N} \Psi^{2}(0) .
$$

We will show below that $\Psi(0)$ is exponentially suppressed

$$
\Psi^{2}(0) \propto \exp \left(-M_{0} B\right)
$$

If the hight of the barrier is bigger than inverse transverse size of the brane, then the suppression coefficient is substantial. Therefore, we discover that the coupling of the lowest mode to an ordinary matter is exponentially suppressed, whereas it's mass is only power sensitive to the width of the brane

$$
m_{0}^{2} \propto \frac{M_{0}^{2} B}{R} .
$$

\footnotetext{
${ }^{3}$ In this case we think of the brane as a sort of "kink" for certain field theory.
} 
Therefore, this force can be exponentially weaker than gravity. This effect has important phenomenological implications.

Let us discuss the dilaton-mediated forces at scales $r<<R$. The interaction is mediated by the whole tower of the corresponding KK states. We should compare this interaction with the gravitational potential at the same distances. The question is whether the resulting potential is still suppressed after the summation of all the KK modes are performed. To find this out consider a simple toy model in which the brane in compact space is approximated by a periodic array of square potential barriers. This is a good model for a kink with nonzero thickness. In the approximation of a very high barrier the wave function takes the form:

$$
\left|\Psi_{m_{n}}(y=0)\right|^{2}=\mathcal{G}\left(m_{n}, R\right) \exp \left(-M_{0} B\right)
$$

where

$$
m_{n}^{2}=\frac{n^{2}}{R^{2}}+m_{0}^{2}, \quad \mathcal{G}\left(m_{n}, R\right) \propto \frac{m_{n} \sin ^{2}\left(m_{n} R\right)(R+B)}{R m_{n}-2 \sin \left(2 m_{n} R\right)}
$$

and the zero-mode mass is defined as $m_{0}^{2}=M_{0}^{2} B / R$. This demonstrates that wave functions for all KK states are exponentially suppressed as $\exp \left(-M_{0} B\right)$. Furthermore, the effective interaction constants of these states with the localized matter are exponentially suppressed as well.

Finally, we need to determine the power-low-force which is produced due to the exchange of all these modes. The problem simplifies computationally if one considers the approximation of a single potential barrier with the width $B$ in infinite extra space. In this case, the potential produced by the tower of the KK states reads as follows:

$$
V(r) \sim \frac{m_{1} m_{2} G_{5} \exp (-M B)}{r^{4} M^{2}}+\ldots
$$

Therefore, the same exponential suppression of the force mediated by the dilaton.

Phenomenologically, the most interesting for the sub-millimeter gravitational measurements is the case of two or more extra dimensions. Here the same qualitative features hold as far as the exponential sensitivity is concerned. Generically, the mass of the dilaton is

$$
m_{0}^{2} \propto M_{0}^{2} \frac{B^{N}}{R^{N}}
$$

whereas the wave function of the light modes is suppressed as $\sim \exp \left(-M_{0} B\right)$.

All the results obtained above for the dilaton are equally applicable to the bulk gauge fields which acquire masses due to the Higgs effect on our brane (this Higgs effect should not be confused with the Standard Model Higgs effect.). Therefore, the potential mediated by the exchange of the bulk gauge fields can exhibit the similar behavior:

(I) At distances $r>>\langle\chi\rangle^{-1}(\chi$ - being the additional Higgs field giving masses to the bulk gauge bosons ) they can be parametrically (exponentially) suppressed compared to gravity; 
(II) At distances $\langle\chi\rangle^{-1}<<r<<R$ they can exhibit distinctive (from gravity) dependence on $r$ in the force law.

Finally, there might be subtleties related to the couplings of the longitudinal components of gauge fields. Let us address this issue. Since we study the gauge fields which acquire masses via the Higgs effect on the "fat" brane, the would be Goldstone boson (which becomes a longitudinal component of the massive gauge field) is not a bulk mode. Therefore, one might naively expect that the coupling of this mode to the ordinary fermions is suppressed only by the relative thickness of the "fat" brane. This is certainly true if there is a direct coupling between the standard model fermions and the Higgs field $\chi$ which breaks a bulk gauge symmetry, and, moreover, its interactions, as, for instance,

$$
\chi \bar{\Psi} \Psi,
$$

are not invariant under independent $U_{B}(1)$ global transformations of $\chi$ t. If the terms similar to one in (38) are absent, there is no direct coupling of the Goldstone mode to ordinary fermions. Indeed, the corresponding Higgs field could couple to fermions through invariant couplings such as $\chi^{*} \chi \bar{\Psi} \Psi$. In the gauge-less limit Goldstone bosons would decouple. Thus, in this case, the only possible coupling to the longitudinal components of the gauge fields would be through their transverse components, which as we have demonstrated above, are exponentially suppressed. On the other hand, the terms of the form (38) would give rise to a direct coupling of the standard model particles to a low-scale Goldstone boson. There are severe astrophysical bounds on such couplings, which would require a scale of the symmetry breaking to be at least $10^{10} \mathrm{GeV}$ or so. Therefore, the phenomenological constraints force us to the scenario in which the matter fermions have no direct couplings to the Goldstone mode and, thus, to the longitudinal component of the bulk gauge fields.

\section{Conclusions}

In conclusion, we have shown that in many cases bulk fields, such as a dilaton or gauge fields, have parametrically suppressed interactions with ordinary matter on a brane. These fields mediate forces which can be much weaker than what is expected naively. This fact should be taken into account while deriving possible experimental bounds on masses of the bulk states from the sub-millimeter gravitational experiments [3, 国.

\section{Acknowledgments}

We are grateful to Zurab Berezhiani and Marko Kolanovic for useful discussions.

\footnotetext{
${ }^{4}$ Note that if the bulk symmetry in question is used to suppress dangerous operators (e.g., baryon number violating terms), such couplings are not desirable and must be suppressed by some discrete symmetries. This is an additional motivation for not having them in the theory. However, for the sake of generality, we include these terms into the consideration.
} 
The work of G.D. is supported in part by a David and Lucille Packard Foundation Fellowship for Science and Engineering. G.G. is supported by NSF grant PHY-9423002. M.P. is supported in part by NSF grant PHY-9722083.

\section{References}

[1] N. Arkani-Hamed, S. Dimopoulos, G. Dvali, Phys. Lett. B429, 263 (1998); I. Antoniadis, N. Arkani-Hamed, S. Dimopoulos, G. Dvali, Phys. Lett. B436, 257 (1998) .

[2] N. Arkani-Hamed, S. Dimopoulos, G. Dvali, Phys. Rev. D59, 0860 (1999) .

[3] J.C. Price, in Proceedings of International Symposium on Experimental Gravitational Physics, ed. P.F. Michelson, Guangzhou, China (World Scientific, Singapore, 1988); J.C. Price et al., NSF Proposal, 1996; J. Long, "Laboratory Search for Extra-Dimensional Effects in the Sub-millimeter Regime", Talk given at the International Conference on Physics Beyond Four Dimensions, ICTP, Trieste, Italy; July 3-6, (2000).

[4] A. Kapitulnik, T. Kenny, NSF Proposal, 1997 ;

A. Kapitulnik, "Experimental Tests of Gravity Below $1 \mathrm{~mm}$ ", Talk given at the International Conference on Physics Beyond Four Dimensions, ICTP, Trieste, Italy; July 3-6, (2000).

[5] G. Dvali, G. Gabadadze, M. Porrati, Phys. Lett. B485, 208 (2000). 